\title{
Schizophrenia and offspring's risk for adverse pregnancy outcomes and infant death
}

\author{
Emma Nilsson, Christina M. Hultman, Sven Cnattingius, Petra Otterblad Olausson, Camilla Björk \\ and Paul Lichtenstein
}

\section{Background}

Women with schizophrenia are at increased risk for adverse pregnancy outcomes. It is not known whether offspring born to fathers with schizophrenia also have an increased risk.

\section{Aims \\ To evaluate paternal and maternal influences on the association between schizophrenia and pregnancy outcomes. \\ Method \\ A record linkage including 2 million births was made using swedish population-based registers. The risk for adverse pregnancy outcomes was evaluated through logistic regression.}

\section{Results}

Offspring with a mother or father with schizophrenia faced a doubled risk of infant mortality, which could not be explained by maternal behaviour alone during pregnancy. Excess infant death risk was largely attributable to post-neonatal death. Maternal factors (e.g. smoking) explained most of the other risks of adverse pregnancy outcomes among both mothers and fathers with schizophrenia.

\section{Conclusions}

The risks to offspring whose fathers had schizophrenia suggest that, in addition to maternal risk behaviour, nonoptimal social and/or parenting circumstances are of importance.

\section{Declaration of interest}

None. Funding detailed in Acknowledgements.
Women with schizophrenia have an increased risk for adverse pregnancy outcomes. ${ }^{1-4}$ We have previously shown that the increased risk of having a low-birth-weight infant among women with schizophrenia decreased from $80 \%$ to $30 \%$ when maternal factors such as smoking, low education and single motherhood were controlled for. ${ }^{5}$ Nevertheless, an increased risk for low birth weight remained even after these covariates were controlled for.

The offspring's risk for adverse pregnancy outcomes if the father has schizophrenia is less well known. A recent study by King-Hele et al found an increased risk for sudden infant death syndrome among both offspring to mothers and fathers with schizophrenia. ${ }^{6}$ Furthermore, a study by Webb et al found an increased risk for post-neonatal death among offspring whose fathers had schizophrenia. ${ }^{7}$ However, the risk was not statistically significant in either of these studies and a meta-analysis by Webb et al indicated a need for evidence concerning the effects of exposure to paternal disorder. ${ }^{3}$

We investigated the association between schizophrenia in mothers and fathers and the risk for four adverse pregnancy outcomes: infant death, low birth weight, pre-term delivery, and small for gestational age in a large population-based Swedish cohort.

\section{Method}

\section{Study design}

The Multi-Generation Register contains information on children born in Sweden since 1932 and their parents. ${ }^{8}$ This register was used to gather information on parents and their siblings. The Medical Birth Register includes prospectively collected information from standardised medical records on virtually all births in Sweden from 1973 onwards. ${ }^{9,10}$

The Hospital Discharge Register provides data on hospital discharges and diagnoses as determined by the treating physician. The information on psychiatric care is in essence complete since $1973{ }^{11}$ The diagnoses in the Birth and Discharge Registers are coded according to the ICD (ICD-8 up to 1986, according to ICD-9 from 1987 through 1996, and finally according to ICD-10 from 1997 and onwards). ${ }^{12,13,14}$ The Cause of Death Register includes information about date of death and underlying cause of death, coded according to the ICD classifications. The population-based Education Register was used to gather information on the parents' highest level of formal education by 31 December 2001. ${ }^{8}$

The registers were linked through the unique national registration number assigned to each Swedish resident. ${ }^{15}$ The study population was defined by women delivering singleton births from 1983 (since information on maternal smoking is available from 1983 ) through to 2002 ( $n=1890550)$. Thus, as compared with our previous study, ${ }^{5}$ this study comprised updated information from the Medical Birth Register and Hospital Discharge Register, and also information from the Multi-Generation Register on the infants' father.

\section{Measures}

\section{Adverse pregnancy outcomes}

Infant death was defined as a death within the first year of life. We defined neonatal death as death from 0 to 27 completed days of life, and post-neonatal death as death from 28 to 364 days. Causes of infant death were grouped into seven categories (congenital conditions, aspyhxia-related conditions, immaturity-related conditions, infections, sudden infant death syndrome (SIDS), external causes, and remaining causes) according to the International Collaborative Effort on Perinatal and Infant Mortality (ICD-codes are included in the online supplement Appendix DS1). ${ }^{16}$ Other outcome variables were low birth weight $(<2500 \mathrm{~g})$, pre-term delivery $(\leqslant 36$ completed weeks $)$ and small-for-gestational-age births (birth weight more than 2 standard deviations below the mean birth weight for gestational age, according to the Swedish fetal growth curve). ${ }^{17}$ Gestational age was based on ultrasound measurements in the second trimester. 
If this information was missing, gestational length was calculated from the last menstruation.

\section{Schizophrenia}

Individuals with schizophrenia were defined as those with at least one hospital admission for schizophrenia (ICD-8 and ICD-9 code 295 and ICD-10 codes F20, F21, F23.1, F23.2, and F25) during the period of 1977-2002. We included information on schizophrenia of mothers, fathers, and the parents' full siblings and half-siblings.

\section{Covariates}

At the first visit to antenatal clinic, we obtained the information on maternal smoking (categorised as non-daily smoking, one to nine cigarettes per day, or at least ten cigarettes per day) and cohabitation status (defined as living with the infant's father or not). Information about maternal age, parity and complications during pregnancy or delivery was obtained from obstetrical records. Maternal and paternal age was defined as age in completed years at the time of delivery $(\leqslant 24,25-29,30-34$ or $\geqslant 35$ years), and parity as the number of births ( $1,2-3$ or $\geqslant 4$ births). Information about the parents' highest level of formal education was grouped into $\leqslant 9,10-11,12,13-14$ or $\geqslant 15$ years.

\section{Statistical analyses}

Evaluation of the distribution of the covariates was performed using the chi-squared method. Multivariate analyses were performed including the independent variable in logistic regression analysis. The risk for adverse pregnancy outcomes for mothers or fathers with schizophrenia was calculated using odds ratio (OR) and 95\% confidence interval (CI) and for this we used Generalized Estimation Equation models (GEE). This method makes use of all cases and allows a large sample of controls and simultaneously controls for dependency induced by multiple deliveries of a mother. In these analyses, we used all relatives with schizophrenia and random samples of 300000 births.
To investigate whether the risks for adverse pregnancy outcomes were higher for women with schizophrenia compared with fathers with schizophrenia, we tested whether the interaction effect between the gender of the parent and schizophrenia was significant $(P<0.05)$. Statistical analyses were performed using the SAS System version 8.

\section{Results}

Compared with women and men without schizophrenia, parents with schizophrenia tended to have higher maternal and paternal age $(P<0.001)$, to be more likely to be having their first infant $(P<0.001)$ and to have spent fewer years in education $(P<0.001)$. Women with schizophrenia were less likely to cohabit with the infant's father, and fathers with schizophrenia were less likely to cohabit with their pregnant spouse $(P<0.001)$. Women with schizophrenia were more likely to smoke during pregnancy $(P<0.001)$ (Table 1$)$.

Table 2 shows the distribution of mothers and fathers with and without schizophrenia and the relation to infant mortality. Compared with offspring of mothers without schizophrenia, offspring of mothers with schizophrenia had a more than a two-fold increase in risk for infant death (crude $O R=2.4$ ). Similarly, compared with offspring of fathers without schizophrenia, offspring of fathers with schizophrenia had an increased risk for infant death (crude $\mathrm{OR}=1.9$ ). Thus, schizophrenia in both fathers and mothers seems to confer a risk for infant death. The difference in risk for infant death for mothers compared with fathers with schizophrenia was not significant $(P=0.20)$.

Next, adjustments were made for covariates known to influence the association between schizophrenia and infant death (schizophrenia in spouse, maternal age, parity, maternal and paternal education, cohabitation status and maternal smoking). After adjustment, the risks for infant death slightly decreased, but were still significantly elevated (Table 2 ).

The association between both maternal and paternal schizophrenia and infant death remained after controlling for the covariates. This could be because of environmental causes, but it could

\begin{tabular}{|c|c|c|c|c|}
\hline & \multicolumn{2}{|c|}{ Mother with schizophrenia } & \multicolumn{2}{|c|}{ Father with schizophrenia } \\
\hline & Yes $(n=3119), \%$ & No $(n=1880976), \%$ & Yes ( $n=2829), \%$ & No $(n=1865434), \%$ \\
\hline \multicolumn{5}{|c|}{ Parental age, years } \\
\hline$\leqslant 24$ & 22.3 & 23.1 & 13.2 & 11.0 \\
\hline $25-29$ & 31.6 & 36.7 & 27.3 & 30.2 \\
\hline 30-34 & 27.1 & 27.4 & 27.6 & 31.9 \\
\hline$\geqslant 35$ & 19.1 & 12.8 & 31.9 & 26.9 \\
\hline \multicolumn{5}{|l|}{ Parity } \\
\hline 1 & 46.2 & 41.9 & 23.9 & 12.1 \\
\hline $2-3$ & 45.3 & 51.4 & 57.8 & 71.4 \\
\hline 4 & 8.4 & 6.7 & 18.2 & 16.5 \\
\hline \multicolumn{5}{|c|}{ Education, years } \\
\hline$\leqslant 9$ & 28.1 & 12.9 & 28.3 & 18.0 \\
\hline $10-11$ & 40.2 & 35.8 & 41.3 & 38.8 \\
\hline 12 & 11.1 & 17.5 & 12.8 & 13.6 \\
\hline $13-14$ & 12.5 & 17.0 & 8.9 & 13.9 \\
\hline$\geqslant 15$ & 8.0 & 16.7 & 8.7 & 15.7 \\
\hline \multicolumn{5}{|c|}{ Cohabitation } \\
\hline Yes & 79.2 & 94.6 & 84.1 & 95.0 \\
\hline No & 20.8 & 5.4 & 15.9 & 5.0 \\
\hline \multicolumn{5}{|c|}{ Maternal smoking, cigarettes/day } \\
\hline None & 52.1 & 78.1 & & \\
\hline $1-9$ & 23.1 & 13.8 & & \\
\hline$\geqslant 10$ & 24.9 & 8.1 & & \\
\hline
\end{tabular}




\begin{tabular}{|c|c|c|c|c|c|c|c|}
\hline & \multirow[b]{3}{*}{ Schizophrenia } & \multirow[b]{3}{*}{$n$} & \multirow[b]{3}{*}{$\%$} & \multicolumn{4}{|c|}{ Risk of mortality } \\
\hline & & & & \multicolumn{2}{|c|}{ Crude } & \multicolumn{2}{|c|}{ Adjusted $^{\mathrm{a}}$} \\
\hline & & & & OR & $\mathrm{Cl}$ & OR & $\mathrm{Cl}$ \\
\hline \multicolumn{8}{|c|}{ Infant mortality (0-364 days) } \\
\hline \multirow[t]{2}{*}{ Mother } & Yes & 34 & 1.1 & 2.4 & $1.7-3.4$ & 2.2 & $1.5-3.2$ \\
\hline & No & 8365 & 0.4 & Ref. & & Ref. & \\
\hline \multirow[t]{2}{*}{ Father } & Yes & 25 & 0.9 & 1.9 & $1.2-2.8$ & 1.8 & $1.1-2.8$ \\
\hline & No & 8374 & 0.5 & Ref. & & Ref. & \\
\hline \multicolumn{8}{|c|}{ Neonatal mortality (0-27 days) } \\
\hline \multirow{2}{*}{ Mother } & Yes & 15 & 0.5 & 1.8 & $1.1-3.0$ & - & \\
\hline & No & 5156 & 0.3 & Ref. & & & \\
\hline \multirow[t]{2}{*}{ Father } & Yes & 12 & 0.4 & 1.6 & $0.9-2.8$ & - & \\
\hline & No & 5159 & 0.3 & Ref. & & & \\
\hline \multicolumn{8}{|c|}{ Post-neonatal mortality (28-364 days) } \\
\hline \multirow{2}{*}{ Mother } & Yes & 19 & 0.6 & 3.5 & $2.2-5.6$ & - & \\
\hline & No & 3209 & 0.2 & Ref. & & & \\
\hline \multirow[t]{2}{*}{ Father } & Yes & 13 & 0.5 & 2.7 & $1.6-4.7$ & - & \\
\hline & No & 3215 & 0.2 & Ref. & & & \\
\hline
\end{tabular}

also be because of a common genetic effect. To illuminate whether a genetic factor contributes to this association, we studied the risk for infant death among parental full and half-siblings to individuals with schizophrenia. A genetic factor would be indicated if the risk was significantly higher for parental full siblings compared with parental half-siblings. However, the risk for infant death if the parent had a sibling with schizophrenia was not increased (data not shown).

In additional analyses on time of death (Table 2), offspring of mothers with schizophrenia had a more than a three-fold increased risk for post-neonatal death $(\mathrm{OR}=3.5)$ as compared with an almost two-fold increased risk of neonatal death $(\mathrm{OR}=1.8)$. The same pattern was shown for offspring of fathers with schizophrenia $(\mathrm{OR}=2.7$ and 1.6 respectively). Due to the rarity of the outcome, multivariate analyses of neonatal and post-neonatal death were not computable.

To analyse if the distribution of causes of death differed between infants without a parent with schizophrenia as compared with infants with a mother or father with schizophrenia, we categorised the causes of death into seven subgroups (Table 3). No substantial differences in the distribution were found between offspring of individuals with and without schizophrenia. Two of the infants who died within the first year of life had both a mother and a father with schizophrenia. The causes of death for these infants were 'intrauterine hypoxia and birth asphyxia' and 'respiratory distress syndrome'.

Table 4 shows the distribution of mothers and fathers with and without schizophrenia and the relation to low birth weight, preterm delivery and small-for-gestational-age births. Mothers with schizophrenia had a significantly increased risk of giving birth to a low-birth-weight infant (crude $\mathrm{OR}=1.6$ ). The risk was also significantly increased for partners of fathers with schizophrenia. The difference in risk for low-birth-weight offspring among mothers compared with fathers with schizophrenia were close to significance $(P=0.06)$. After adjustment for the covariates, these risks were no longer increased (Table 4 ).

Compared with mothers without schizophrenia, mothers with schizophrenia had a significantly increased risk for pre-term delivery (crude $\mathrm{OR}=1.6$ ), while the corresponding risk among offspring of fathers with schizophrenia was minor (crude $\mathrm{OR}=1.1$ ). The risk for pre-term delivery among mothers with schizophrenia was significantly higher compared with the risk for spouses of fathers with schizophrenia $(P=0.01)$. After adjustments for the covariates, the risk for pre-term delivery among mothers was attenuated and of borderline significance, while the risk for fathers reached unity (Table 4).

Both mothers and fathers with schizophrenia had an increased risk for having a small-for-gestational-age infant. No statistically

Table 3 Causes of death during the first year of life among infants of mothers and fathers with and without schizophrenia

\begin{tabular}{|c|c|c|c|c|c|c|c|c|}
\hline \multirow[b]{3}{*}{ Cause of death } & \multicolumn{4}{|c|}{ Schizophrenia in mother } & \multicolumn{4}{|c|}{ Schizophrenia in father } \\
\hline & \multicolumn{2}{|c|}{ Yes } & \multicolumn{2}{|c|}{ No } & \multicolumn{2}{|c|}{ Yes } & \multicolumn{2}{|c|}{ No } \\
\hline & $n$ & (\%) & $n$ & (\%) & $n$ & (\%) & $n$ & (\%) \\
\hline Congenital conditions & 13 & (38) & 3449 & (41) & 11 & (44) & 3451 & (41) \\
\hline Sudden infant death syndrome & 4 & $(12)$ & 1306 & (16) & 6 & (24) & 1304 & (16) \\
\hline Asphyxia-related conditions & 3 & (9) & 758 & (9) & 2 & (8) & 759 & (9) \\
\hline Immaturity-related conditions & 7 & (21) & 1479 & (18) & 3 & $(12)$ & 1483 & (18) \\
\hline Infections & 3 & (9) & 873 & (10) & 0 & (0) & 876 & (10) \\
\hline External causes & 1 & (3) & 128 & (2) & 2 & (8) & 127 & (2) \\
\hline Remaining causes & 3 & (9) & 372 & (4) & 1 & (4) & 374 & (4) \\
\hline
\end{tabular}




\begin{tabular}{|c|c|c|c|c|c|c|}
\hline & \multirow[b]{3}{*}{$n$} & \multirow[b]{3}{*}{$\%$} & \multicolumn{4}{|c|}{ Risk for adverse pregnancy outcomes } \\
\hline & & & \multicolumn{2}{|c|}{ Crude } & \multicolumn{2}{|c|}{ Adjusted $^{\mathrm{a}}$} \\
\hline & & & OR & $95 \% \mathrm{Cl}$ & OR & $95 \% \mathrm{Cl}$ \\
\hline \multicolumn{7}{|c|}{ Low birth weight } \\
\hline \multicolumn{7}{|c|}{ Schizophrenia in mother } \\
\hline Yes & 174 & 5.6 & 1.6 & \multirow[t]{2}{*}{$1.4-1.9$} & 1.1 & \multirow[t]{2}{*}{$0.9-1.4$} \\
\hline No & 63144 & 3.4 & Ref. & & Ref. & \\
\hline \multicolumn{7}{|c|}{ Schizophrenia in father } \\
\hline Yes & 125 & 4.5 & 1.3 & \multirow[t]{2}{*}{$1.1-1.6$} & 1.1 & \multirow[t]{2}{*}{$0.9-1.3$} \\
\hline No & 63193 & 3.4 & Ref. & & Ref. & \\
\hline \multicolumn{7}{|c|}{ Pre-term delivery } \\
\hline \multicolumn{7}{|c|}{ Schizophrenia in mother } \\
\hline Yes & 198 & 6.4 & 1.6 & \multirow[t]{2}{*}{$1.4-1.8$} & 1.2 & \multirow[t]{2}{*}{$1.0-1.4$} \\
\hline No & 76725 & 4.1 & Ref. & & Ref. & \\
\hline \multicolumn{7}{|c|}{ Schizophrenia in father } \\
\hline Yes & 132 & 4.7 & 1.1 & \multirow[t]{2}{*}{$1.0-1.4$} & 1.0 & \multirow[t]{2}{*}{$0.8-1.2$} \\
\hline No & 76791 & 4.1 & Ref. & & Ref. & \\
\hline \multicolumn{7}{|c|}{ Small for gestational age } \\
\hline \multicolumn{7}{|c|}{ Schizophrenia in mother } \\
\hline Yes & 121 & 3.9 & 1.4 & \multirow[t]{2}{*}{$1.2-1.7$} & 0.9 & \multirow[t]{2}{*}{$0.7-1.2$} \\
\hline No & 49304 & 2.6 & Ref. & & Ref. & \\
\hline \multicolumn{7}{|c|}{ Schizophrenia in father } \\
\hline Yes & 116 & 4.1 & 1.5 & \multirow[t]{2}{*}{$1.3-1.9$} & 1.2 & \multirow[t]{2}{*}{$1.0-1.6$} \\
\hline No & 49309 & 2.6 & Ref. & & Ref. & \\
\hline
\end{tabular}

significant difference between maternal and paternal risk was observed $(P=1.0)$. The covariates explained the increased risk for small-for-gestational-age births of mothers with schizophrenia, whereas a moderately increased risk remained for fathers with schizophrenia (Table 4).

\section{Discussion}

We found that offspring of mothers and fathers with schizophrenia faced a substantial increase in risk for infant mortality, which cannot be explained by included covariates. In line with previous studies, this study confirmed that women with schizophrenia are at increased risk for adverse pregnancy outcomes (i.e. low birth weight, pre-term birth and small for gestational age $)^{1-3}$, but these risks were generally no longer increased after adjustments for a number of covariates known to be more prevalent among women with schizophrenia.

Previous studies have found that women with schizophrenia often smoke, misuse other substances and are socio-economically disadvantaged. ${ }^{18}$ These variables are well-known risk factors for adverse pregnancy outcomes and smoking is causally related to foetal growth retardation and possibly also to stillbirth and preterm birth. ${ }^{19}$ In contrast to most previous studies, we were able to control for some of these confounders. Except for infant death, most of the increased risk for adverse pregnancy outcomes among parents with schizophrenia was accounted for by the covariates (e.g. smoking, single motherhood, and maternal and paternal education). In the adjusted analyses, there remained a small increased risk of pre-term birth among infants of mothers with schizophrenia. Since a large number of social and lifestyle-related factors influence risk of pre-term birth, ${ }^{20}$ we find it likely that this small risk increase may be due to residual confounding of unmeasured socially related risk factors.

We found an increased risk for infant death if the father had schizophrenia even after adjusting for covariates. The significantly increased risks for both mothers and fathers with schizophrenia suggest that the risk for infant death could not be explained only by maternal behaviour during pregnancy. Moreover, in line with previous studies, we found an increased risk for post-neonatal death among infants with a mother or father with schizophrenia, possibly indicating that parental neglect, or perhaps extreme social disadvantage, may be of importance. ${ }^{21}$ Categorisation of causes of death did not further clarify the increased risk for infant death among parents with schizophrenia.

In contrast to a meta-analysis by Sacker et $a l^{2}$ on studies conducted before 1995, we could show that offspring of fathers with schizophrenia had increased risks for infant death, low birth weight, and being small for gestational age. Our study is much larger than previous studies, and we find it likely that paternal schizophrenia also confers a risk for adverse pregnancy outcomes. None the less, since the risks related to outcomes other than infant mortality were largely explained when covariates were included in the models, we agree with Sacker et al, that adverse pregnancy outcomes are probably not a consequence of schizophrenia in the parents, but rather a consequence of the adverse socio-environmental conditions. The risk for infant death if a parent had a sibling with schizophrenia was not increased, indicating that the association may not be explained by genetic effects.

Although the current study first and foremost is directed towards understanding the aetiology of adverse pregnancy outcome in schizophrenia, our results may also be discussed within a frame of general policies on how to improve provision of support and early detection of possible complications. At the individual level, antenatal care is today most often attentive to earlier psychiatric symptoms and vulnerability of the mother, but likely less focused on paternal characteristics. These results, coupled with research showing that offspring of parents with psychiatric disorders are at a higher risk of death from all causes over the lifespan, warrant a closer liaison between neonatal, paediatric and psychiatric health professionals. 


\section{Methodological considerations}

This study fulfils several methodological criteria proposed for studies of the association between schizophrenia and adverse pregnancy outcomes. ${ }^{1,3,22}$ The study population is based on an entire population birth cohort, the group was relatively unaffected by migration, and the data were recorded and collected from prospectively filed paediatric records. Furthermore, the dependence that occurs when a woman has more than one delivery was taken into account. This study includes complete population-based registers of births and hospital discharges with generally considered high validity and reliability. ${ }^{9,23}$

A limitation of this register-based study is that we did not have information on a number of factors that could have influenced the risk for adverse pregnancy outcomes among parents with schizophrenia. We were not able to control for the potential confounding of maternal medication during pregnancy, alcohol/ drug misuse, infections during pregnancy or attachment problems due to an exacerbation of specific symptoms of schizophrenia (e.g. social cognitive and emotion processing deficits) during the postpartum period. Nevertheless, since both mothers and fathers with schizophrenia had an increased risk for adverse pregnancy outcomes, it is not likely that maternal medication or other unmeasured maternal factors during pregnancy could explain all the excess risk. Furthermore, this study lacked information on maternal support and state during pregnancy and post-partum as well as maternal health support. This information could have further illuminated the associations. Another limitation is that the study is register-based and therefore information on social and economic factors, other than maternal education, was not available. Thus, we have not been able to evaluate the effect of, for example, impoverishment. To broaden the understanding of the increased risk for adverse pregnancy outcomes, symptom-based subgroups (e.g. hallucinations, paranoia etc.) of parents with schizophrenia should be analysed in future studies.

In summary, we have shown an increased risk for adverse pregnancy outcomes among offspring of fathers as well as mothers with schizophrenia. This increased risk is primarily explained by the pregnant women's psychosocial situation such as smoking and single motherhood. The increased risks for infant death among offspring of fathers with schizophrenia suggest that improved awareness in maternity and child care units of the risk factors following a pregnancy to parents with schizophrenia is warranted.

\footnotetext{
Emma Nilsson, PhD, Department of Medical Epidemiology and Biostatistics, Karolinska Institutet, and Centre for Epidemiology, National Board of Health and Welfare, Stockholm; Christina M. Hultman, PsyD, Department of Medical Epidemiology and Biostatistics, Karolinska Institutet, Stockholm, and Department of Neuroscience, Psychiatry, Ulleråker, Uppsala University, Uppsala; Sven Cnattingius, MD, PhD, Department of Medical Epidemiology and Biostatistics, Karolinska Institutet, Stockholm; Petra Otterblad Olausson, PhD, Centre for Epidemiology, National Board of Health and Welfare, Stockholm; Camilla Björk, MSc, Department of Medica Evidemiology and Biostatistics, Karolinska Institutet, Stockholm; Paul Lichtenstein, PhD, Department of Medical Epidemiology and Biostatistics, Karolinska Institutet, PhD, Department of
Stockholm, Sweden.

Correspondence: Emma Nilsson, Centre for Epidemiology, National Board of Health and Welfare, SE-106 30 Stockholm, Sweden. Email: Emma.Nilsson@Socialstyrelsen.se

First received 14 Sep 2007, final revision 18 Apr 2008, accepted 15 May 2008
}

\section{Acknowledgements}

This study was supported by the Swedish Council for Working Life and Social Research (grant no. 2013/2002) and the Swedish Research Council (grant no. K2004-21X-15084-01A.

\section{References}

1 Jablensky AV, Morgan V, Zubrick SR, Bower C, Yellachich LA. Pregnancy delivery, and neonatal complications in a population cohort of women with schizophrenia and major affective disorders. Am J Psychiatry 2005; 162: 7991.

2 Sacker A, Done DJ, Crow TJ. Obstetric complications in children born to parents with schizophrenia: a meta-analysis of case-control studies. Psychol Med 1996; 26: 279-87.

3 Webb R, Abel K, Pickles A, Appleby L. Mortality in offspring of parents with psychotic disorders: a critical review and meta-analysis. Am J Psychiatry 2005; 162: 1045-56.

4 Bennedsen BE, Mortensen PB, Olesen AV, Henriksen TB, Frydenberg M. Obstetric complications in women with schizophrenia. Schizophr Res 2001; 47: $167-75$.

5 Nilsson $\mathrm{E}$, Lichtenstein $\mathrm{P}$, Cnattingius $\mathrm{S}$, Murray RM, Hultman $\mathrm{CM}$. Women with schizophrenia: pregnancy outcome and infant death among their offspring. Schizophr Res 2002; 58: 221-9.

6 King-Hele SA, Abel KM, Webb RT, Mortensen PB, Appleby L, Pickles AR. Risk of sudden infant death syndrome with parental mental illness. Arch Gen Psychiatry 2007; 64: 1323-30.

7 Webb RT, Abel KM, Pickles AR, Appleby L, King-Hele SA, Mortensen PB. Mortality risk among offspring of psychiatric inpatients: a population-based follow-up to early adulthood. Am J Psychiatry 2006; 163: 2170-7.

8 Statistics Sweden. Educational attainment of the population 2001. Official statistics of Sweden UF 37 SM 0101, 2001: (http://www.scb.se/templates/ Publikation_44633.asp)

9 Cnattingius S, Ericson A, Gunnarskog J, Kallen B. A quality study of a medical birth registry. Scand J Soc Med 1990; 18: 143-8.

10 Centre for Epidemiology. The Swedish Medical Birth Register - A Summary of Content and Quality. Research Report 2003. (http://www.socialstyrelsen.se/ NR/rdonlyres/E9BE4DDE-95EE-4E3F-A56F-36CA5125CA8C/1132/20031123.pdf).

11 Centre for Epidemiology. The Swedish Hospital Discharge Register. 2005 (http://www.socialstyrelsen.se/en/Statistics/statsbysubject/The+Swedish+ Hospital+Discharge+Register.htm)

12 World Health Organization. International Classification of Diseases: Manual of the International Statistical Classification of Diseases, Injuries and Causes of Death (ICD-8). WHO, 1967.

13 World Health Organization. International Classification of Diseases: Manual of the International Statistical Classification of Diseases, Injuries and Causes of Death (ICD-9). WHO, 1978

14 World Health Organization. International Classification of Diseases: Manual of the International Statistical Classification of Diseases, Injuries and Causes of Death (ICD-10). WHO, 1992.

15 Lunde AS, Lundeborg S, Lettenstrom GS, Thygesen L, Huebner J. The personnumber systems of Sweden, Norway, Denmark, and Israel. Vital Health Stat 1980; 2: $1-59$

16 Cole S, Hartford RB, Bergsjo P, McCarthy B. International collaborative effort (ICE) on birth weight, plurality, perinatal, and infant mortality. III: a method of grouping underlying causes of infant death to aid international comparisons. Acta Obstet Gynecol Scand 1989; 68: 113-7.

17 Marsal K, Persson PH, Larsen T, Lilja H, Selbing A, Sultan B. Intrauterine growth curves based on ultrasonically estimated foetal weights. Acta Paediatr 1996; 85: 843-8.

18 Bennedsen BE, Mortensen PB, Olesen AV, Henriksen TB. Preterm birth and intra-uterine growth retardation among children of women with schizophrenia. Br J Psychiatry 1999; 175: 239-45.

19 Cnattingius S. The epidemiology of smoking during pregnancy: smoking prevalence, maternal characteristics, and pregnancy outcomes. Nicotine Tob Res 2004; 6 (suppl 2): S125-40.

20 Goldenberg RL, Culhane JF, lams JD, Romero R. Epidemiology and causes of preterm birth. Lancet 2008; 371: 75-84.

21 Bennedsen BE, Mortensen PB, Olesen AV, Henriksen TB. Congenital malformations, stillbirths, and infant deaths among children of women with schizophrenia. Arch Gen Psychiatry 2001; 58: 674-9.

22 McNeil TF. Perinatal risk factors and schizophrenia: selective review and methodological concerns. Epidemiol Rev 1995; 17: 107-12.

23 Ekholm B, Ekholm A, Adolfsson R, Vares M, Osby U, Sedvall GC, Jonsson EG. Evaluation of diagnostic procedures in Swedish patients with schizophrenia and related psychoses. Nordic J Psychiatry 2005; 59 457-64. 